\title{
Multiple Ionization Effects in X-Ray Emission Induced by Heavy Ions
}

\author{
M. Czarnota, M. Pajek, D. Banaś, \\ Institute of Physics, Świętokrzyska Academy, 25-406 Kielce, Poland
}

J.-Cl. Dousse, Y.-P. Maillard, O. Mauron, P. A. Raboud, M. Berset,

Physics Department, University of Fribourg, CH-1700 Fribourg, Switzerland

D. Chmielewska, J. Rzadkiewicz, Z. Sujkowski,

Sottan Institute for Nuclear Studies, 05-400 Otwock-Świerk, Poland

J. Hoszowska,

European Synchrotron Radiation Facility (ESRF), F-38043 Grenoble, France

K. Słabkowska, and M. Polasik

Faculty of Chemistry, Nicholas Copernicus University, 87-100 Toruń, Poland

Received on 29 July, 2005

\begin{abstract}
The $\mathrm{x}$-ray satellite structure of $\mathrm{Pd} \mathrm{L}_{1,2}\left(\mathrm{~L}_{3} \mathrm{M}_{4,5}\right)$ transition excited by an impact of $\mathrm{O}^{7+}$ and $\mathrm{Ne}^{6+}$ ions with energies 279 and $178 \mathrm{MeV}$, respectively, which were measured using a high-resolution von Hamos crystal spectrometer, is discussed in terms of the multi-configuration Dirac-Fock (MCDF) calculations. We demonstrate, by using the arguments of the general central limit theorem (GCLT), that a structure of complex M-shell satellites of Pd $\mathrm{L} \alpha_{1,2}\left(\mathrm{M}^{-m}\right)$ transitions for a higher number of spectator vacancies $(m>4)$, which consists of hundreds of thousands of individual x-ray transitions as obtained from the MCDF calculations, can be well described by a single Voigtian profile. The Lorentzian width of such Voigtian line can be well modeled by using the results of the MCDF calculations for simpler configurations with a number of vacancies $m \leq 4$. This method allows one to describe realistically a complex structure of M-shell satellites, thus extending the applicability of the MCDF calculations, which are limited by an increasing complexity of numerical calculations.
\end{abstract}

Keywords: Multiple ionization; X-ray satellite structure; MCDF calculations

\section{INTRODUCTION}

The x-rays emitted from atoms multiply ionized by heavy ions exhibit, apart from the well known X-ray diagram lines, the satellite structure corresponding to different multi-vacancy configurations present at the moment of the x-ray emission. High-resolution measurements of excited x-ray satellites give thus access to study the structure of multi-vacancy configurations in atoms. However, in order to extract from such complicated spectra the $\mathrm{x}$-ray transitions the structure of $\mathrm{x}$ ray multiplets and their Lorentzian widths for a given multivacancy configuration as well as the experimental Gaussian broadening have to be known. This results from the fact that for heavy ion impact the x-ray spectra, containing X-ray satellites with up to several spectator vacancies in the inner-shells, become extremely complex and, consequently, cannot be fitted uniquely without performing the MCDF calculations of the structure of individual $\mathrm{x}$-ray multiplets and realistic modelling of their widths. In this paper we discuss a new method of analysis of complex x-ray spectra which, using the general central limit theorem arguments, describes the complex x-ray satellites as the smooth Vogtian profile having known mean value and width, which can be obtained from MCDF calculations. This method, which is based on our earlier works $[1,2]$, is presently extended to high-resolution spectroscopy. An alternative approach of analysis of x-ray spectra emitted from multiply ionized atoms can be found in a recent work by Hor- vat et al. [3].

In this paper we discuss the $\mathrm{M}$ - and $\mathrm{N}$-shell satellites of $\mathrm{Pd}$ $\mathrm{L}_{1,2}\left(\mathrm{~L}_{3} \mathrm{M}_{4,5}\right) \mathrm{x}$-ray transitions excited by fast $\mathrm{O}^{7+}$ and $\mathrm{Ne}^{6+}$ ions [4], which were measured with high-resolution $(\sim 1$ $\mathrm{eV}$ ) using crystal diffraction spectrometer [5]. The measured $\mathrm{X}$-ray spectra were compared with predictions of the multiconfiguration Dirac-Fock (MCDF) calculations. The details concerning the MCDF calculations adopted here are described in Ref. [6]. In fact, the x-ray satellites of the $L \alpha_{1,2}\left(\mathrm{~L}_{3} \mathrm{M}_{4,5}\right)$ transitions which are dominated by a small number of $\mathrm{M}$-shell satellites, as for instance for $\mathrm{O}^{7+}$ ion impact on palladium $(m \leq 4)$, can be well reproduced by MCDF calculations (see Fig. 1). However, for more complex configurations with a higher number of spectator vacancies $(m>4)$ the MCDF calculations become too complex numerically to be performed in practice. This is the case of $\mathrm{Ne}^{6+}$ impact on palladium (see Fig. 2), for which up to $m=7 \mathrm{M}$-shell satellites have to be calculated in order to reproduce the measured x-ray spectrum of $\mathrm{Pd} \mathrm{L} \alpha_{1,2}\left(\mathrm{~L}_{3} \mathrm{M}_{4,5}\right)$. We demonstrate, by using the general central limit theorem arguments, that the M-shell satellites for a higher number of spectator vacancies $(m>4)$ can be approximated by a single Voigtian profile for which a mean energy and width can be obtained by extrapolating the MCDF calculations for configurations with a smaller number of spectator vacancies. 


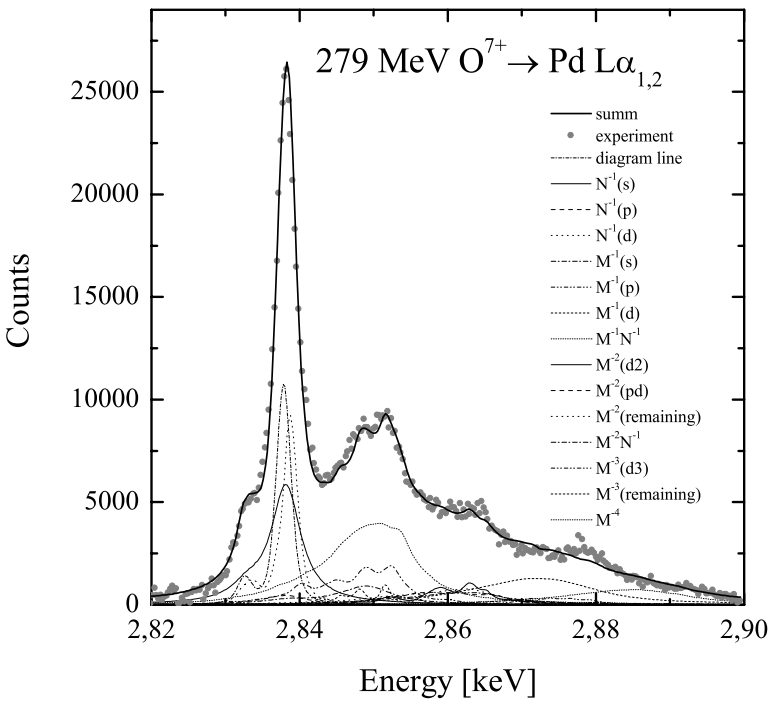

FIG. 1: Measured x-ray satellite structure for $L \alpha_{1,2}$ transition in palladium excited by $279 \mathrm{MeV} \mathrm{O}^{7+}$ ions. The data are compared with the predictions of the MCDF calculations including up to $m=4$ spectator vacancies in the $\mathrm{M}$ - and $\mathrm{N}$-shells. The individual components of the calculated spectrum are marked in the figure.

\section{EXPERIMENT}

The high-resolution measurements of the M- and N-shell satellites of $\mathrm{Pd} \mathrm{L \alpha}_{1,2}\left(\mathrm{~L}_{3} \mathrm{M}_{4,5}\right) \mathrm{x}$-ray transitions excited by fast $\mathrm{O}^{7+}$ and $\mathrm{Ne}^{6+}$ ions have been performed [8] at the Philips cyclotron in the Paul Scherrer Institute (PSI) in Villigen, Switzerland, using a von Hamos high-resolution diffraction spectrometer [5]. The $\mathrm{x}$-ray spectra of $\mathrm{L} \alpha_{1,2}\left(\mathrm{~L}_{3} \mathrm{M}_{4,5}\right)$ transitions were excited by $\mathrm{O}^{7+}$ and $\mathrm{Ne}^{6+}$ ion beams of energies 279 and $178 \mathrm{MeV}$, respectively, bombarding thin metallic palladium foils. The x-rays were measured by means of a highresolution von Hamos spectrometer [5] with a precision of about $1 \mathrm{eV}$ for studied Pd L-x-rays $(\sim 3 \mathrm{keV})$, including an experimental Gaussian resolution of about $0.7 \mathrm{eV}$. The von Hamos spectrometer was equipped with a quartz $(1 \overline{1} 1)$ crystal curved with a radius of $25.4 \mathrm{~cm}$. The $\mathrm{x}$-rays were measured with the CCD detector covering in one setting the $\mathrm{x}$-ray energy range of about $50 \mathrm{eV}$. Consequently, the x-ray spectra of Pd $\mathrm{L} \alpha_{1,2}\left(\mathrm{~L}_{3} \mathrm{M}_{4,5}\right)$ transitions were measured for several settings of the spectrometer. The energy calibration of the spectrometer has been performed by measuring well resolved $\mathrm{K} \alpha_{1,2}$ $\mathrm{x}$-ray lines of vanadium excited by photons from $\mathrm{x}$-ray tube with $\mathrm{Cr}$ anode.

\section{RESULTS AND DISCUSSION}

In order to interpret quantitatively the measured $\mathrm{x}$-ray satellite structure of $\mathrm{Pd} \mathrm{L}_{1,2}\left(\mathrm{~L}_{3} \mathrm{M}_{4,5}\right)$ transitions excited by $\mathrm{Ne}^{6+}$ ions of energy $178 \mathrm{MeV}$, MCDF calculations involving up to seven M-shell spectator vacancies are needed. However, the MCDF calculations for multi-vacancy $\operatorname{Pd} \operatorname{L} \alpha_{1,2}\left(\mathrm{M}^{-m}\right)$ con-

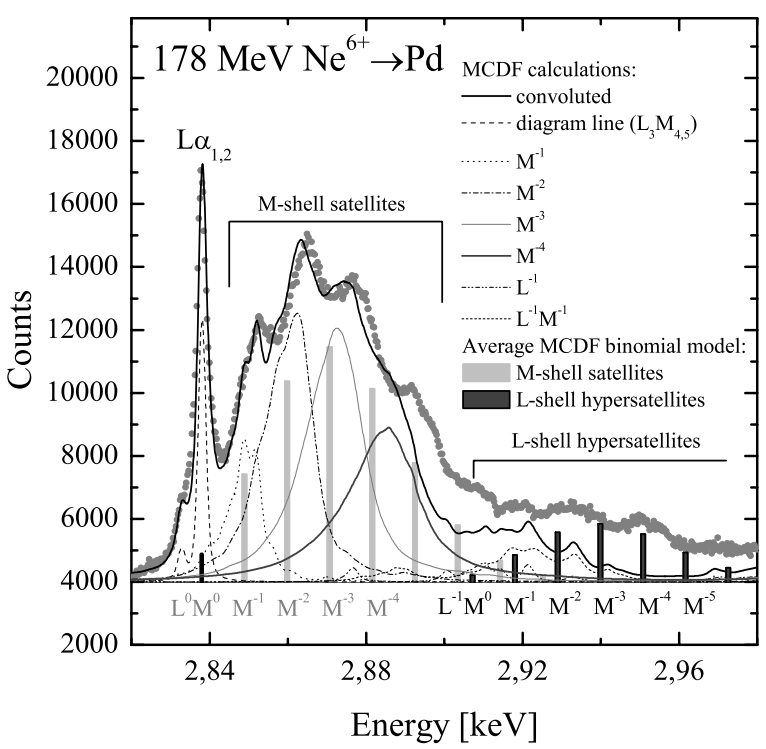

FIG. 2: Measured x-ray satellite structure for $L \alpha_{1,2}$ transition in palladium excited by $178 \mathrm{MeV} \mathrm{Ne}^{6+}$ ions. The data are compared with the predictions of the MCDF calculations including up to $m=4$ spectator vacancies in the $\mathrm{M}$ - and $\mathrm{N}$-shells and the average MCDF binomial model indicating the importance of configurations with up to $m=8$ vacancies in the M-shell as well as the $\mathrm{L}$-shell hypersatellite structure.

figurations become, in practice, numerically intractable for $m>4$. For instance, the MCDF calculations for palladium for $\mathrm{M}^{-4}$ configuration contain 244953 transitions (see Fig. 3) and for mixed $\mathrm{M}^{-1} \mathrm{~N}^{-2}$ configuration 268210 transitions. Such numerical limitation of the applicability of the MCDF calculations asks for developing of alternative approximate methods to treat the complex satellite structures of $\mathrm{x}$-rays excited in ion-atom collisions.

Following the idea presented in our earlier works (see Refs. [1] and [2]) on multiple ionization effects in ion-induced $x-$ ray spectra we suggest that the $\mathrm{x}$-ray profile for complex $\mathrm{x}$ ray transitions can be well approximated by an effective single profile resulting from a convolution of natural Lorentzian and experimental Gaussian widths applied to the calculated MCDF x-ray multiplets consisting of large number of transitions. This observation is based on the firm ground of the general central limit theorem (see Ref. [7]) suggesting a Voigtian type profile as the limiting distribution in our case.

In order to verify this idea, the calculated MCDF structure of $\mathrm{x}$-ray transitions for $\mathrm{Pd} \mathrm{L} \alpha_{1,2}\left(\mathrm{M}^{-m}\right)$ configuration, convoluted with natural Lorentzian widths of individual transitions, assumed to scale approximately with a number of spectator vacancies $m$ as $\Gamma(m)=\Gamma(0)+2 \Gamma_{\text {spec }} \cdot m$, and experimental Gaussian widths of about $0.7 \mathrm{eV}$, are shown in Fig. 4. The effective widths of the resulting Voigtian profiles for complex $\mathrm{x}$-ray multiplets are expected to follow approximately a simple scaling rule, namely,

$$
\Gamma(m)=\Gamma_{M}(0)+\alpha m+\beta \sqrt{m}
$$

where $\alpha$ and $\beta$ are constants which can be fitted for a calcu- 


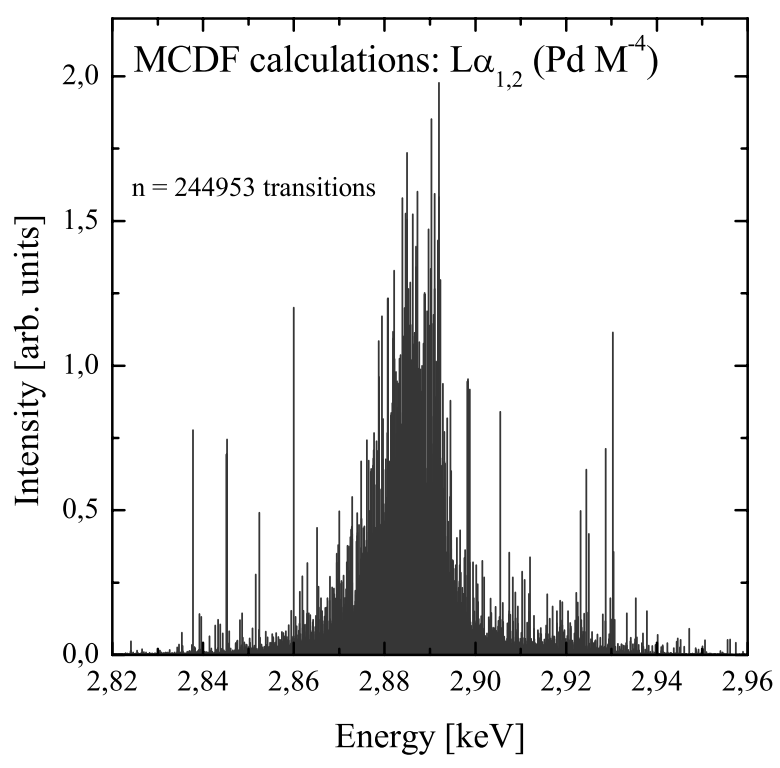

FIG. 3: The structure of $P d L \alpha_{1,2}\left(M^{-4}\right)$ x-ray transitions obtained by using the MCDF calculations.

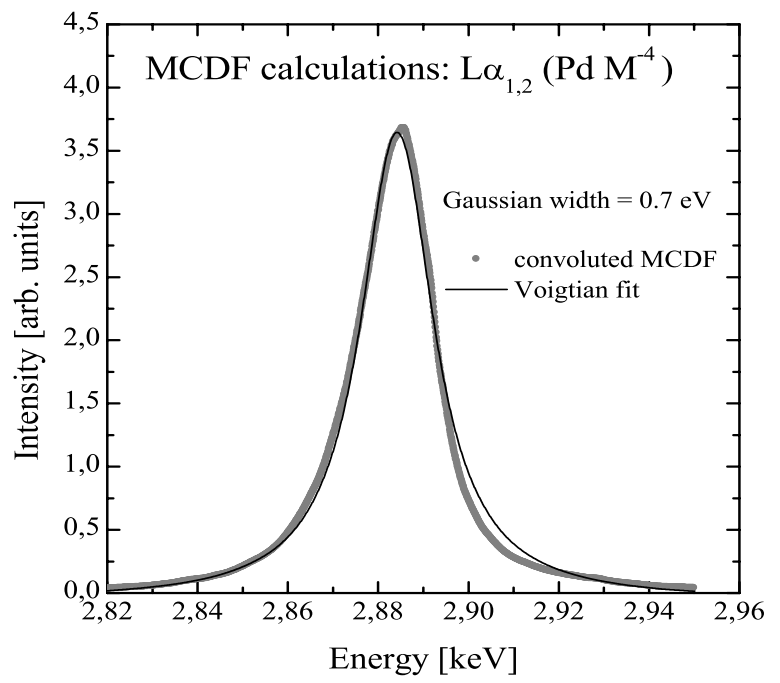

FIG. 4: A profile of $\mathrm{L} \alpha_{1,2}\left(\mathrm{M}^{-4}\right) \mathrm{x}$-ray transitions, as obtained by convolution of calculated MCDF transitions with natural Lorentzian (see text) and experimental Gaussian widths, fitted by a single Voigtian profile. Note the smooth shape of the calculated (MCDF) profile and the reasonable good fit by a Voigtian.

lated MCDF configuration with a smaller number of spectator vacancies, $m \leq 4$ in our case. This formula, which uses once more the arguments of the GCLT theorem, has been derived by summing up the natural width $\Gamma(m)$ of the Lorentzian distribution of x-ray transition energy and the width of the binomial distribution of a number of vacancies randomly distributed in the M-shell. In fact, the variance of the binomial distribution $\sigma^{2}(m)=N_{M} p_{M}\left(1-p_{M}\right)$, where $N_{M}$ is a number of electrons in the M-shell, scales for $p_{M}=m / M \ll 1$ as $\sigma^{2}(m) \propto m$ yielding the following approximate estimate for a width: $\Gamma_{b i n}(m) \propto \sqrt{m}$.

The fitted effective widths of x-ray transitions for $\mathbf{M}^{-m}$ configurations, shown in Fig. 5, fully justify the model assumed. Consequently, such a parameterization of Voigtian widths for complex multi-vacancy configurations, combined with a known linear parameterization of their mean energies adopted in the average MCDF binomial model (Ref. [6]) of $\mathrm{x}$-ray satellite structure, allows one to describe in a realistic way the complex x-ray spectra excited by heavy ions, which include much more spectator vacancies than can be treated numerically in an exact way using the MCDF calculations.

The present findings open a possibility to describe a complex satellite structure of $\operatorname{Pd} L \alpha_{1,2}\left(\mathrm{~L}_{3} \mathrm{M}_{4,5}\right)$ transitions such as in the discussed $\mathrm{x}$-ray spectra excited by $\mathrm{Ne}^{6+}$ ions of energy $178 \mathrm{MeV}$ exhibiting M- and N-shell satellites. However, a final interpretation of such X-ray spectra needs further MCDF calculations for the observed L-shell hypersatellite structure overlapping with M-shell satellites. Such MCDF calculations of L-shell hypersatellites are in progress.

\section{CONCLUSIONS}

A novel approximate description of x-ray spectra for complex multi-vacancy $\mathbf{M}^{-m}$ configurations has been proposed, which is based on the general central limit theorem. The calculated profiles of $\mathrm{L} \alpha_{1,2}\left(\mathrm{M}^{-m}\right) \mathrm{x}$-ray transitions in palladium are well described by the proposed model. Complex multivacancy configurations, involving up to about ten spectator

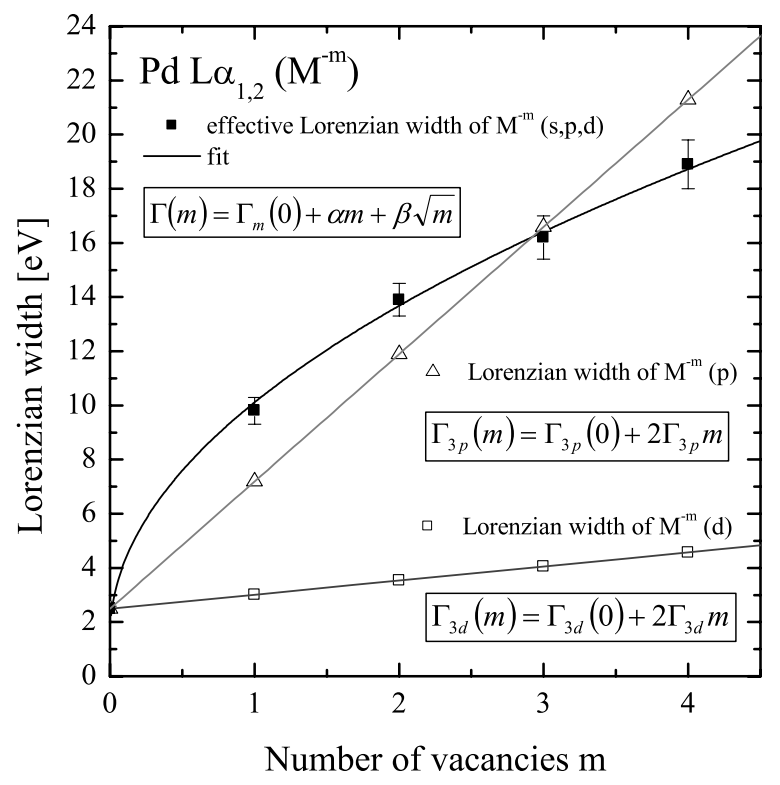

FIG. 5: Fitted effective Lorentzian widths of $P d L \alpha_{1,2}\left(M^{-m}\right)$ MCDF $\mathrm{x}$-ray transitions for spectator vacancies in s-, p-, and d-states, which are well fitted by the approximate formula $\Gamma(m)=\Gamma_{M}(0)+\alpha m+$ $\beta \sqrt{m}$. Assumed natural widths for $\mathrm{M}^{-m}$ vacancy configurations with vacancies in $3 p$ and $3 d$ states are also shown in the figure. 
vacancies, can be treated within this approach, which significantly extends the applicability of the MCDF calculations for describing multiple ionization effects in X-ray spectra excited by heavy ions.
This work was supported by the Polish State Committee for Scientific Research under the Grant No. 1P0301326 and the Swiss National Science Foundation.

\section{Acknowledgments}

[1] D. Banaś et al., Nucl. Instrum. and Methods B 195, 233 (2002).

[2] M. Pajek, D. Banás, J. Semaniak, J. Braziewicz et al., Phys. Rev. A 68, 022705 (2003)

[3] V. Horvat et al., Phys. Rev. A 71, 062709 (2005).

[4] M. Czarnota et al., Nucl. Instrum. and Methods B 205, 133(2003).

[5] J. Hoszowska et al., Nucl. Instrum. and Methods A 376, 129
(1996).

[6] M. Polasik, Phys. Rev. A 52, 227 (1995).

[7] G. Samorodnitsky and M.S. Taqqu, Stable Non-Gaussian Random Processes, Chapman\&Hall, New York, 1994.

[8] M. Czarnota et al., Rad. Phys. Chem. 68, 121 (2003). 